This article is published with open access at Springerlink.com

\title{
On the Nilpotency Class of a Generalized 3-Abelian Group
}

\author{
Bounabi Daoud*, Meriem Hamitouche and Khalissa Merikhi
}

\begin{abstract}
A group $G$ is called 3-abelian if the map $x \mapsto x^{3}$ is an endomorphism of $G$ and it is called generalized 3-abelian, if there exist elements $c_{1}, c_{2}, c_{3} \in G$ such that the map $\varphi: x \longmapsto x^{c_{1}} x^{c_{2}} x^{c_{3}}$ is an endomorphism of $G$. Abdollahi, Daoud and Endimioni have proved that a generalized 3-abelian group $G$ is nilpotent of class at most 10 . Here, we improve the bound to 3 and we show that the exponent of its derived subgoup is finite and divides 9. We also prove that $G$ is 3-Levi, 9-central, 9-abelian and 3-nilpotent of class at most 2.
\end{abstract}

Mathematics Subject Classification (2010). Primary 20E36; Secondary $20 \mathrm{~F} 18$.

Keywords. Generalized 3-abelian group, nilpotent, 3-nilpotent.

\section{Introduction and Results}

Let $n \geq 2$ be an integer. A group $G$ is called $n$-abelian whenever $(x y)^{n}=$ $x^{n} y^{n}$ for all $x, y \in G$; or equivalently the map $x \mapsto x^{n}$ is an endomorphism of $G$. Levi [7] has proved that a group $G$ is 3 -abelian if and only if it is 2Engel and the exponent of its derived subgroup $[G, G]$ divides 3. Trotter [10] has proved that a 3 -abelian group $G$ is abelian whenever the map $x \mapsto x^{3}$ is an automorphism of $G$. A group $G$ is called generalized $n$-abelian whenever there exist elements $c_{1}, \ldots, c_{n} \in G$ such that the map $x \mapsto x^{c_{1}} \ldots x^{c_{n}}$ is an endomorphism of $G$. The class of generalized $n$-abelian groups is closed under the formation images, and finite direct products. Obviously, every $n$-abelian group is generalized $n$-abelian and it is easy to see that every generalized 2-abelian group is abelian. It is clear that by conjugating we may assume one of $c_{1}, \ldots, c_{n}$ to be the trivial element.

Abdollahi, Daoud and Endimioni [1, Theorem 3.1] have proved that a generalized 3-abelian group $G$ is nilpotent of class at most 10, and abelian

${ }^{*}$ Corresponding author. 
whenever the map $x \mapsto x^{c_{1}} x^{c_{2}} x^{c_{3}}$ is a monomorphism of $G$ or the center $\zeta(G)$ has no element of order 3 . Here we improve the previous results as following.

Theorem 1.1. Let $G$ be a generalized 3-abelian group. Then

i) $G$ is nilpotent of class at most 3 .

ii) The exponent of $[G, G]$ divides 9 and the exponent of $\gamma_{3}(G)$ divides 3 .

The free 4-generator group of exponent 3 is clearly a 3 -abelian group and it is well known that it is nilpotent of class 3 . Hence the bound 3 for the class of generalized 3-abelian groups is sharp.

Now for any non-zero integer $n$, a group $G$ is called $n$-Levi if $\left[x, y^{n}\right]=$ $[x, y]^{n}$ for all $x, y \in G$. It is called $n$-central if $n \geq 1$ and $\left[x, y^{n}\right]=1$ for all $x, y \in G$. We show that there is a relation beween generalized 3-abelian groups $G$ and $n$-Levi or $n$-central groups.

Theorem 1.2. Let $G$ be a generalized 3-abelian group admitting an endomorphism of the form

$$
\phi: x \longmapsto x^{a} x x^{b}
$$

for some $a, b \in G$. Then

i) $G$ is 3-Levi, 9-central and 9-abelian.

ii) The subgroup Im $\phi$ is abelian. In particular, if $\phi$ is injective or surjective, $G$ is abelian.

Let $m \neq 0$ be an integer. Baer [2] introduced the $m$-center of a group $G$ as follows:

$$
\mathcal{Z}(G, m)=\left\{a \in G \mid(a x)^{m}=a^{m} x^{m} \text { and }(x a)^{m}=x^{m} a^{m} \text { for all } x \in G\right\} .
$$

The set $\mathcal{Z}(G, m)$ is a characteristic subgroup of $G$ for any non-zero integer $m$. L.-C. Kappe and M. L. Newel [5] proved that

$$
(a x)^{m}=a^{m} x^{m} \text { for all } x \in G \Longleftrightarrow(x a)^{m}=x^{m} a^{m} \text { for all } x \in G .
$$

Thus only one of the $m$-commutativity conditions suffices to define the $m$ center $\mathcal{Z}(G, m)$. If $m$ is a positive integer, the upper $m$-central series $\mathcal{Z}_{i}(G, m)$ is defined inductively as the following: $\mathcal{Z}_{0}(G, m)=1, \mathcal{Z}_{1}(G, m)=\mathcal{Z}(G, m)$ and $\mathcal{Z}_{i+1}(G, m) / \mathcal{Z}_{i}(G, m)=\mathcal{Z}\left(G / \mathcal{Z}_{i}(G, m), m\right)$ for $i \geq 1$.

We then get an ascending series.

$$
1=\mathcal{Z}_{0}(G, m) \leq \mathcal{Z}_{1}(G, m) \leq \cdots \leq \mathcal{Z}_{i}(G, m) \leq \mathcal{Z}_{i+1}(G, m) \leq \ldots
$$

A group $G$ is said to be $m$-nilpotent of class at most $k$, if $\mathcal{Z}_{k}(G, m)=G$. Karasev [6] intoduced the $m$-derived subgroup of a group $G$ as follows: For a given integer $m \geq 2$ and two elements $x, y$ of $G$, the $m$-commutator of $x$ and $y$ is defined by $[x, y]_{m}=(x y)^{m} y^{-m} x^{-m}$. The $m$-derived subgroup of $G$ is then the subgroup generated by the set of the $m$-commutators of $G$. It is a fully invariant subgroup of $G$ and is denoted by $[G, G]_{m}$. If $H$ is a normal subgroup of $G$, then $G / H$ is $m$-abelian if and only if $[G, G]_{m} \leq H . G /[G, G]$ being abelian, $[G, G]_{m} \leq[G, G]$. We have the following theorem:

Theorem 1.3. Every generalized 3-abelian group is 3-nilpotent of class at most 2. Moreover the exponent of its 3-derived subgroup divides 3 . 


\section{Proofs}

Notations used in this paper are standard. For a group $G$ and the elements $x_{1}$, $x_{2}, \ldots, x_{n}, x, y \in G$, the commutators $\left[x_{1}, x_{2}, \ldots, x_{n}\right]$ and $\left[x_{n}, y\right]$ are defined inductively by the rules:

$$
\left[x_{1}, x_{2}\right]=x_{1}^{-1} x_{2}^{-1} x_{1} x_{2},\left[x_{1}, y\right]=[x, y]=x^{-1} x^{y}
$$

and for $n \geq 2\left[x_{1}, x_{2}, \ldots, x_{n+1}\right]=\left[\left[x_{1}, x_{2}, \ldots, x_{n}\right], x_{n+1}\right],\left[x_{n}, y\right]=\left[\left[x_{n-1}, y\right], y\right]$.

For a given integer $i \geq 1$, we denote by $[G, G], \zeta_{i}(G)$ and $\gamma_{3}(G)$ respectively the derived subgroup, the $i$ th-center and the third term of the lower central series of $G$. We denote by $H \leq G$ if $H$ is a subgroup of $G$. To prove Theorems 1.1, 1.2 and 1.3 we need the following lemmas.

Lemma 2.1. Let $G$ be a generalized $n$-abelian group admitting an endomorphism of the form $\psi: x \mapsto x^{c_{1}} \cdots x^{c_{n}}$ where $c_{1}, \ldots, c_{n} \in G$. Then, $G$ is $n$-abelian whenever $c_{1}, \ldots, c_{n} \in \zeta_{2}(G)$.

Proof. Let $x \in G$. As $c_{1}, \ldots, c_{n} \in \zeta_{2}(G)$ we have that $x,\left[x, c_{1}\right], \ldots\left[x, c_{n}\right]$ commute. Thus

$$
x^{\psi}=\prod_{1 \leq i \leq n} x\left[x, c_{i}\right]=x^{n} \prod_{1 \leq i \leq n}\left[x, c_{i}\right] .
$$

Therefore

$$
\begin{gathered}
(x y)^{\psi}=(x y)^{n} \prod_{1 \leq i \leq n}\left[x y, c_{i}\right]=(x y)^{n} \prod_{1 \leq i \leq n}\left[x, c_{i}\right]\left[y, c_{i}\right] \\
x^{\psi} y^{\psi}=x^{n} \prod_{1 \leq i \leq n}\left[x, c_{i}\right] y^{n} \prod_{1 \leq i \leq n}\left[y, c_{i}\right]=x^{n} y^{n} \prod_{1 \leq i \leq n}\left[x, c_{i}\right]\left[y, c_{i}\right]
\end{gathered}
$$

whence $(x y)^{n}=x^{n} y^{n}$.

Lemma 2.2. Let $G$ be a metabelian group, $x, y \in G$ and $u, v \in[G, G]$. Then, for any integer $n \geq 1$, the following assertions hold.

(a) $[u v, x]=[u, x][v, x]$.

(b) $\left[x, y^{n}\right]=\prod_{1 \leq i \leq n}[x, i y]^{\left(\begin{array}{c}n \\ i\end{array}\right)}$.

(c) $\left(x y^{-1}\right)^{n}=x^{n} \prod_{0<i+j<n}[x, i y, j]^{\left(\begin{array}{c}n \\ i+j+1\end{array}\right)} y^{-n}$.

Proof. (a) is easy to prove as $[G, G]$ is abelian. For the proofs of (b) and (c) see [4].

Proof of Theorem 1.1. i) Let $G$ be a generalized 3-abelian group with the given endomorphism $\phi$ defined by $x^{\phi}=x^{a} x x^{b}$ for all $x \in G$, where $a, b \in G$ are fixed. To prove that $G$ is nilpotent of class three, it is enough to show that every 4-generated subgroup $\left\langle g_{1}, g_{2}, g_{3}, g_{4}\right\rangle$ of $G$ is nilpotent of class at most 3. Now $H=\left\langle g_{1}, g_{2}, g_{3}, g_{4}, a, b\right\rangle$ is clearly invariant under the action of $\phi$ and is thus a generalized 3-abelian group as well. We can thus replace $G$ by $H$ and it suffices then to show that $H$ is nilpotent of class at most 3. By [1, Théorème 3.1], $H$ is nilpotent. Now one can use nq package of Werner Nickel [8] implemented in GAP [9] and MAGMA [3] to find the nilpotency class 
of $H$. The package nq has the capability of computing the largest nilpotent quotient (if it exists) of a finitely generated group with finitely many identical relations and finitely many relations. For example, if we want to construct the largest nilpotent quotient of a group $G$ with the following presentation

$$
\begin{array}{r}
\left\langle x_{1}, \ldots, x_{n}\right| r_{1}\left(x_{1}, \ldots, x_{n}\right)=\cdots=r_{m}\left(x_{1}, \ldots, x_{n}\right)=1, \\
\left.w\left(x_{1}, \ldots, x_{n}, y_{1}, \ldots, y_{k}\right)=1\right\rangle,
\end{array}
$$

where $r_{1}, \ldots, r_{m}$ are relations on $x_{1}, \ldots, x_{n}$ and $w\left(x_{1}, \ldots, x_{n}, y_{1}, \ldots, y_{k}\right)=1$ is an identical relation in the group $\left\langle x_{1}, \ldots, x_{n}\right\rangle$, one may apply the following code to use the package nq in GAP:

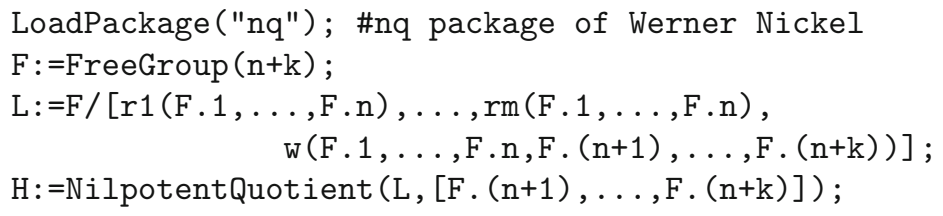

Note that we need to construct the free group of rank $n+k$ because as well as the $n$ generators for $G$ we also have an identical relation with $k$ free variables. Note that the function NilpotentQuotient(L) attempts to compute the largest nilpotent quotient of $\mathrm{L}$ and it will terminate only if $\mathrm{L}$ has a largest nilpotent quotient. Note that our identical relation is $(x y)^{\phi}=x^{\phi} y^{\phi}$ for all $x, y \in G$, which can be written as follows:

$$
(x y)^{a}(x y)(x y)^{b}=\left(x^{a} x x^{b}\right)\left(y^{a} y y^{b}\right)
$$

or

$$
y^{a}(x y) x^{b}\left(x x^{b} y^{a} y\right)^{-1}=1 .
$$

So for our problem we need the following code:

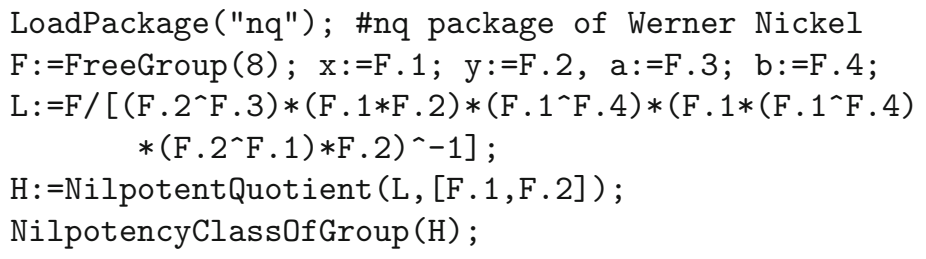

This revealed that the class of $H$ is 3 .

ii) $\phi$ induces on the quotient group $G / \gamma_{3}(G)$ the endomorphism

$$
\bar{x} \longmapsto \bar{x}^{\bar{a}} \bar{x} \bar{x}^{\bar{b}} \text {. }
$$

$G / \gamma_{3}(G)$ is nilpotent of class at most 2 ; by lemma 2.1, it is 3-abelian. By Levi's Theorem, $\exp \left(\left[\frac{G}{\gamma_{3}(G)}, \frac{G}{\gamma_{3}(G)}\right]\right)$, which is equal to $\exp \left(\frac{[G, G] \gamma_{3}(G)}{\gamma_{3}(G)}\right)$, divides 3 . Then $[G, G]^{3} \leq \gamma_{3}(G)(*)$. It follows that $[G, G]^{3} \leq \zeta(G)$ since $\gamma_{3}(G) \leq \zeta(G)$. Now let $x, y, z \in G$.

$$
[x, y, z]^{3}=\left[[x, y]^{3}, z\right]=1
$$


and so $\gamma_{3}(G)^{3}=\{1\}$. From the relation (*), we get $[G, G]^{9} \leq \gamma_{3}(G)^{3}$. Hence $[G, G]^{9}=\{1\}$.

This completes the proof.

Proof of Theorem 1.2. i) Let $x, y \in G$.

$$
\begin{gathered}
{\left[x, y^{3}\right]=\prod_{1 \leq i \leq 3}\left[x,,_{i} y\right]^{\left(\begin{array}{l}
3 \\
i
\end{array}\right)}=[x, y]^{3}[x, y, y]^{3}[x, y, y, y]=[x, y]^{3} .} \\
{\left[x, y^{9}\right]=\prod_{1 \leq i \leq 9}\left[x,,_{i} y\right]^{\left({ }_{i}^{9}\right)}=[x, y]^{9}[x, y, y]^{36} \prod_{3 \leq i \leq 9}\left[x,{ }_{i} y\right]^{\left(\begin{array}{l}
9 \\
i
\end{array}\right)}=1 .} \\
\left(x y^{-1}\right)^{9}=x^{9} \prod_{1 \leq i+j \leq 8}\left[x,{ }_{i} y,{ }_{j} x\right]^{\left({ }_{i+j+1}^{9}\right)} y^{-9} \\
=x^{9}[x, y]^{36}[x, y, x]^{84}[x, y, y]^{84} \prod_{3 \leq i+j \leq 8}\left[x,,_{i} y,,_{j} x\right]^{(\underset{i+j+1}{9})} y^{-9}=x^{9} y^{-9} .
\end{gathered}
$$

ii) Let $x, y \in G$. From part (i) and Theorem 1.1, we know that $G$ is nilpotent of class at most 3 and $\gamma_{3}(G)^{3}=\{1\}$. Therefore

$$
x^{\phi}=x[x, a] x^{2}[x, b]=x^{3}[x, a][x, b][x, a, x]^{2} .
$$

Using furthermore the facts from part (i) and Theorem 1.2 that $[G, G]^{9}=\{1\}$ and that $G$ is 3 -Levi we get

$$
\left[x^{\phi}, y^{\phi}\right]=\left[x^{3}[x, a][x, b], y^{3}[y, a][y, b]\right]=\left[x^{3}, y^{3}\right]=\left[x^{3}, y\right]^{3}=[x, y]^{9}=1 .
$$

This completes the proof.

Proof of Theorem 1.3. We have seen that the quotient group $G / \gamma_{3}(G)$ is 3abelian. So $[G, G]_{3} \leq \gamma_{3}(G)^{(* *)}$ and $\frac{G}{\mathcal{Z}(G, 3)}$ (which is isomorphic to $\frac{\frac{G}{\gamma_{3}(G)}}{\frac{\mathcal{Z}(G, 3)}{\gamma_{3}(G)}}$ ) also is 3 -abelian. Therefore the series

$$
1 \leq \mathcal{Z}(G, 3) \leq G
$$

is 3 -central and $G$ is 3 -nilpotent of class at most 2 . The relation (**) yields $\left([G, G]_{3}\right)^{3} \leq \gamma_{3}(G)^{3}=1$. This completes the proof.

\section{Acknowledgment}

The authors are grateful to the referee for his/her valuable comments and careful readings. The first author thanks Alireza Abdollahi for the help he has provided during his stay at Isfahan University.

\section{References}

[1] A. Abdollahi, B. Daoud and G. Endimioni, Groupes n-abéliens généralisés. Bull. Belg. Math. Soc. 13 (2006), 287-294.

[2] R. Baer, Factorization of n-soluble and n-nilpotent groups. Proc. Amer. Math. Soc. 4 (1953), 15-26.

[3] Wieb Bosma, John Cannon and Catherine Playoust, The Magma algebra system I: The user language. J. Symbolic Comput. 24 (1997), 235-265. 
[4] G. T. Hogan and W. P. Kappe, On the $H_{p}$-problem for finite p-groups. Proc. Amer. Math. Soc. 20 (1969), 450-454.

[5] L. C. Kappe. and M. L. Newell, On the n-centre of a group. In: "Groups - St. Andrews 1989", Vol. 2, 339-352, London Math. Soc. Lecture Note Ser. 160, Cambridge Univ. Press, Cambridge, 1991.

[6] G. A. Karasev, On the theory of n-nilpotent groups. Mat. Zamretki 5 (1969), 653-654.

[7] F. W. Levi, Notes on group theory I, II. J. Indian Math. Soc. (N.S.) 8 (1944), 1-9.

[8] W. Nickel, NQ, 1998, A refereed GAP 4 package. see [9].

[9] The GAP Group, GAP-Groups, Algorithms, and Programming. Version 4.4.12; 2008. Http://www.gap-system.org.

[10] H. F. Trotter, Groups in which raising to a power is an automorphism. Canad. Math. Bull. 8 (1965), 825-827.

Bounabi Daoud, Meriem Hamitouche and Khalissa Merikhi

Department of Mathematics

Laboratory of Fundamental and Numerical Mathematics

Ferhat Abbas University

Setif

Algeria

e-mail: boun_daoud@yahoo.fr

hamitouche85@yahoo.fr

merikhikhalissa@yahoo.fr

Received: May 15, 2012.

Revised: September 30, 2012.

Accepted: November 11, 2012.

Open Access This article is distributed under the terms of the Creative Commons Attribution License which permits any use, distribution, and reproduction in any medium, provided the original author(s) and source are credited. 